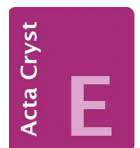

CRYSTALLOGRAPHIC COMMUNICATIONS

ISSN 2056-9890

Received 19 November 202

Accepted 7 January 2022

Edited by J. Reibenspies, Texas A \& M University, USA

Keywords: crystal structure; 2,6-substituted piperidine; hydrogen bonds; DFT.

CCDC reference: 2133146

Supporting information: this article has supporting information at journals.iucr.org/e

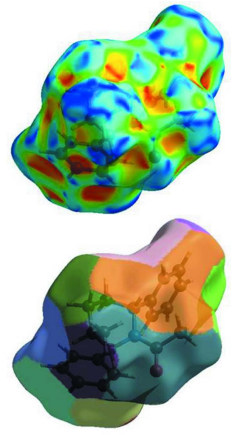

OPEN ๑ ACCESS

\section{Crystal structure, DFT and Hirshfeld surface analysis of $\mathrm{N}$-acetyl-t-3-methyl-r-2,c-6-diphenyl- piperidine}

\author{
P. Periyannan, ${ }^{a}$ M. Beemarao, ${ }^{a}$ K . Karthik, ${ }^{b}$ S. Ponnuswamy ${ }^{b}$ and \\ K. Ravichandran ${ }^{\mathrm{a} *}$
}

aDepartment of Physics, Kandaswami Kandar's College, Velur, Namakkal 638 182, India, and ${ }^{\mathbf{b}} \mathrm{PG}$ and Research' Department of Chemistry, Government Arts College (Autonomous), Coimbatore-641018., Tamil Nadu, India.

*Correspondence e-mail: kravichandran05@gmail.com

In the title compound [systematic name: 1-(3-methyl-2,6-diphenylpiperidin-1yl)ethanone], $\mathrm{C}_{20} \mathrm{H}_{23} \mathrm{NO}$, the piperidine ring adopts a distorted boat conformation, while the phenyl rings subtend a dihedral angle $65.1(2)^{\circ}$. In the crystal, molecules are linked by $\mathrm{C}-\mathrm{H} \cdots \mathrm{O}$ hydrogen bonds into chains extending along the $b$-axis direction. The DFT/B3LYP/6-311 G(d,p) method was used to determine the HOMO-LUMO energy levels. A Hirshfeld surface analysis was conducted to verify the contributions of the different intermolecular interactions, indicating that the important contributions to the crystal packing are from $\mathrm{H} \cdots \mathrm{H}(73.2 \%), \mathrm{C} \cdot \mathrm{H}(18.4 \%)$ and $\mathrm{O} \cdots \mathrm{H}(8.4 \%)$ interactions.

\section{Chemical context}

The structures of a wide array of heterocyclic derivatives have been analysed for their pharma-potentiality over the past three decades (Katritzky, 2010). Among these, derivatives of the six-membered heterocyclic base piperidine have proven to be successful pharmacophores. 2,6-Substituted piperidine derivatives have been found to be useful as tranquilizers and possess a wide range of biological activities such as anti-tumor (Vinaya et al., 2009), antiviral, antimalarial, antibacterial and antifungal activities (Aridoss et al., 2009; Mobio et al., 1989). These have spurred considerable awareness of the synthetic arena based on their structure, reactivity, synthesis and biological properties. We report herein the crystal structure, Hirshfeld surface analysis and DFT computational calculations of the title compound.

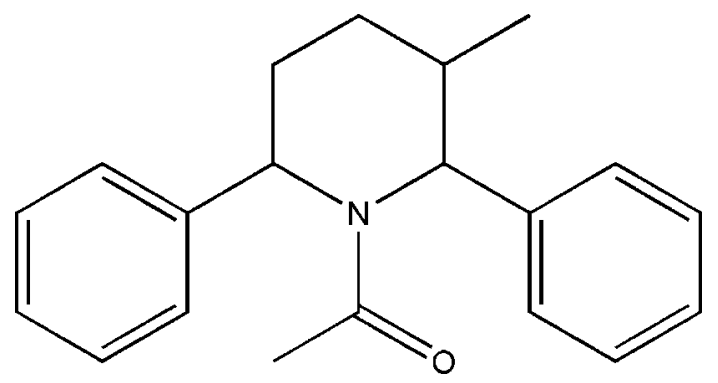

\section{Structural commentary}

The methyl-substituted piperidine title compound crystallizes in the monoclinic space group $P 2_{1}$. A perspective view of the 


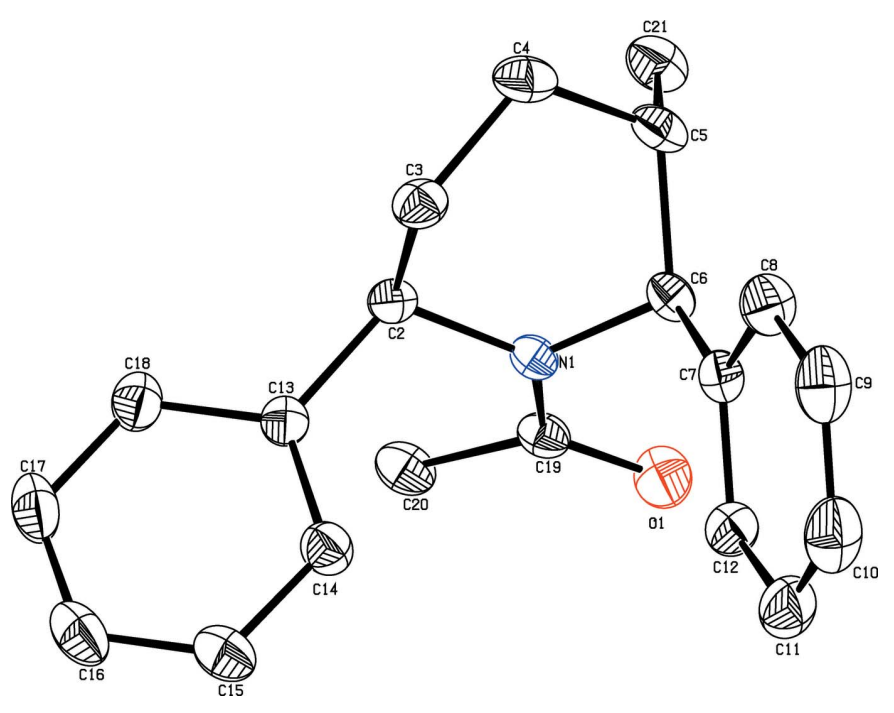

Figure 1

The molecular structure of the title compound, showing the atomic numbering and displacement ellipsoids drawn at the $30 \%$ probability level.

molecule is shown in Fig. 1. The bond lengths and angles are well within the expected limits (Roques et al., 1981), and agree with values observed in related structures (Sekar et al., 1990).

The piperidine ring adopts a distorted boat conformation with puckering parameters (Cremer \& Pople, 1975) and asymmetry parameters (Nardelli, 1983): $q_{2}=0.720$ (2) $\AA, q_{3}=$ $-0.004(3) \AA, \Phi(2)=108.5(2)^{\circ}, \Delta C s(\mathrm{C} 3)$ and $\Delta C s(\mathrm{C} 6)=$ $14.5(2)^{\circ}$, and with maximum deviations of $0.406(3)$ and 0.409 (2) $\AA$, respectively, for atoms C3 and C6 from the best plane of the piperidine ring. The title molecule contains three chiral centres viz., C2, C5 and C6. The absolute configuration of the chiral centres is assigned as $\mathrm{C} 2(R), \mathrm{C} 5(S)$ and $\mathrm{C} 6(S)$. The parent molecule itself is chiral and the configuration cannot be changed during the substitution of acetyl group at the nitrogen.

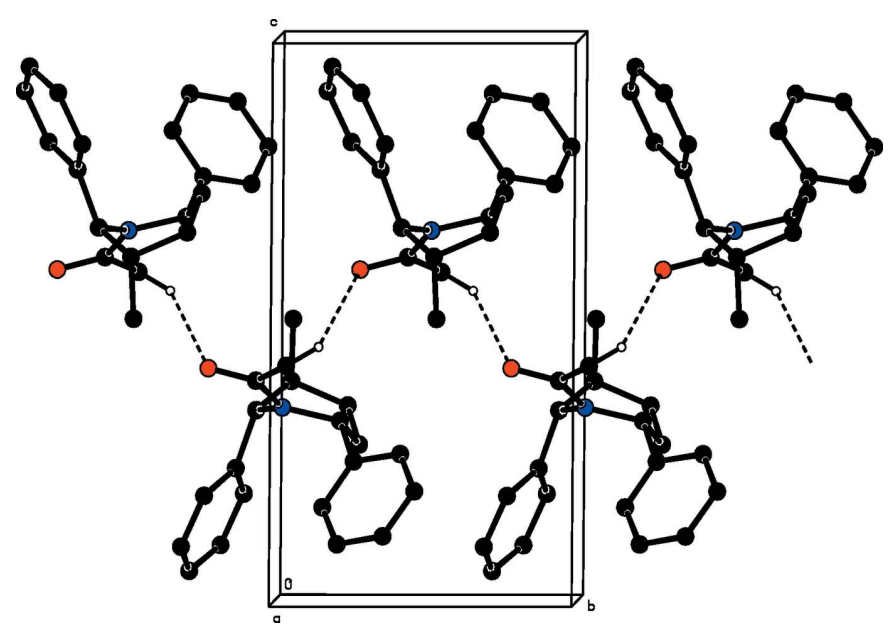

Figure 2

A partial view along the $b$ axis of the crystal packing of the title compound, showing the formation of a molecular chain by $\mathrm{C}-\mathrm{H} \cdots \mathrm{O}$ interactions (dotted lines).
The sum of the bond angles $\left(358.2^{\circ}\right)$ at atom $\mathrm{N} 1$ of the piperidine ring is in accordance with the $s p^{2}$ hybridization state (Beddoes et al., 1986). The phenyl rings at the 2 and 6positions of the piperidine ring occupy equatorial and axial orientations. The corresponding torsion angles are $\mathrm{C} 4-\mathrm{C} 3-$ $\mathrm{C} 2-\mathrm{C} 13=-178.8(2)^{\circ}$ and $\mathrm{C} 4-\mathrm{C} 5-\mathrm{C} 6-\mathrm{C} 7=-74.5(3)^{\circ}$.

The piperidine ring [N1/C2-C6] makes dihedral angles of 82.0 (1) and $58.4(1)^{\circ}$, respectively, with the C13-C18 and C7$\mathrm{C} 12$ phenyl rings, and confirms the fact that the moieties are in axial and equatorial orientations. It is to be noted that there is a possibility of resonance between atoms $\mathrm{N} 1, \mathrm{C} 19$ and $\mathrm{O} 1$ as a result of the delocalization of the hetero $\pi$ electrons of the carbonyl group, which is also confirmed by the torsion angles $\mathrm{C} 2-\mathrm{N} 1-\mathrm{C} 19-\mathrm{O} 1=177.7(2)^{\circ}$ and $\mathrm{C} 6-\mathrm{N} 1-\mathrm{C} 19-\mathrm{O} 1=$ $13.0(3)^{\circ}$.

The methyl group substituted at the 5-position of the piperidine ring is axially oriented, as confirmed by the torsion angles $\mathrm{N} 1-\mathrm{C} 6-\mathrm{C} 5-\mathrm{C} 21=-68.0(3)^{\circ}$ and $\mathrm{C} 3-\mathrm{C} 4-\mathrm{C} 5-$ $\mathrm{C} 21=112.4(3)^{\circ}$, whereas the methyl group substituted at $\mathrm{C} 19$
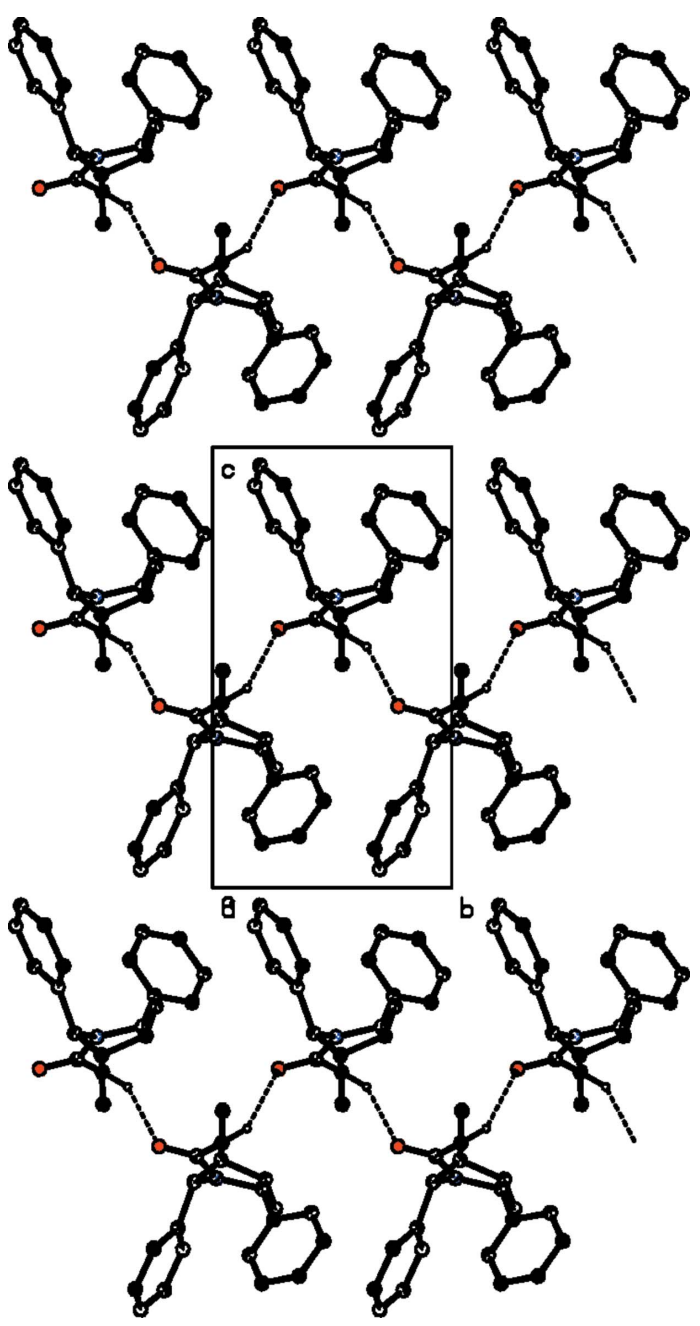

Figure 3

The overall crystal packing of the title compound, viewed along the $b$ axis. Hydrogen bonds are shown as dashed lines, and only the $\mathrm{H}$ atoms involved in hydrogen bonding have been included. 
Table 1

Hydrogen-bond geometry $\left(\AA,^{\circ}\right)$.

\begin{tabular}{lllll}
\hline$D-\mathrm{H} \cdots A$ & $D-\mathrm{H}$ & $\mathrm{H} \cdots A$ & $D \cdots A$ & $D-\mathrm{H} \cdots A$ \\
\hline $\mathrm{C} 20-\mathrm{H} 20 B \cdots \mathrm{O} 1^{\mathrm{i}}$ & 0.96 & 2.44 & $3.292(3)$ & 148 \\
\hline
\end{tabular}

Symmetry code: (i) $-x+1, y+\frac{1}{2},-z+1$.

is oriented equatorially with torsion angle $\mathrm{C} 20-\mathrm{C} 19-\mathrm{N} 1-$ $\mathrm{C} 6=-166.3(2)^{\circ}$ and $\mathrm{C} 20-\mathrm{C} 19-\mathrm{N} 1-\mathrm{C} 2=-1.7(3)^{\circ}$.

\section{Supramolecular features}

The crystal packing features $\mathrm{C}-\mathrm{H} \cdots \mathrm{O}$ interactions (Table 1). Atom $\mathrm{C} 20$ of the molecule at $(x, y, z)$ donates a proton to atom O1 of the molecule at $\left(-x+1, y+\frac{1}{2},-z+1\right)$, forming a $C 4$ zigzag chain (Bernstein et al., 1995) running along the $b$-axis direction as shown in Fig. 2. The overall packing is shown in Fig. 3.

\section{Density functional theory (DFT) study}

The optimized molecular structure and frontier molecular orbitals (FMOs) (Figs. 4 and 5, respectively) were calculated using the DFT/B3LYP/6-311G(d,p) basis set implemented in the GAUSSIAN09 program package (Frisch et al., 2009). The highest occupied molecular orbital (HOMO) and the lowest unoccupied molecular orbital (LUMO) are called frontier molecular orbitals (FMOs) as they lie at the outermost boundaries of the electrons of the molecule. The electron distribution (ED) of the HOMO-1, HOMO, LUMO and LUMO+1 energy levels and the energy values are shown in Fig. 5. The positive and negative phases are represented in green and red, respectively.

The HOMO of the title molecule is localized on one aromatic ring and the $\mathrm{C}=\mathrm{O}$ group, while the $\mathrm{LUMO}$ is located over the whole molecule with the exception of the $\mathrm{CH}_{3}$ group and some carbon and hydrogen atoms in the piperidine ring. Thus the HOMO/LUMO implies an ED transfer to the $\mathrm{C}=\mathrm{O}$ group from the ring. The energy band gap $(\Delta E=$

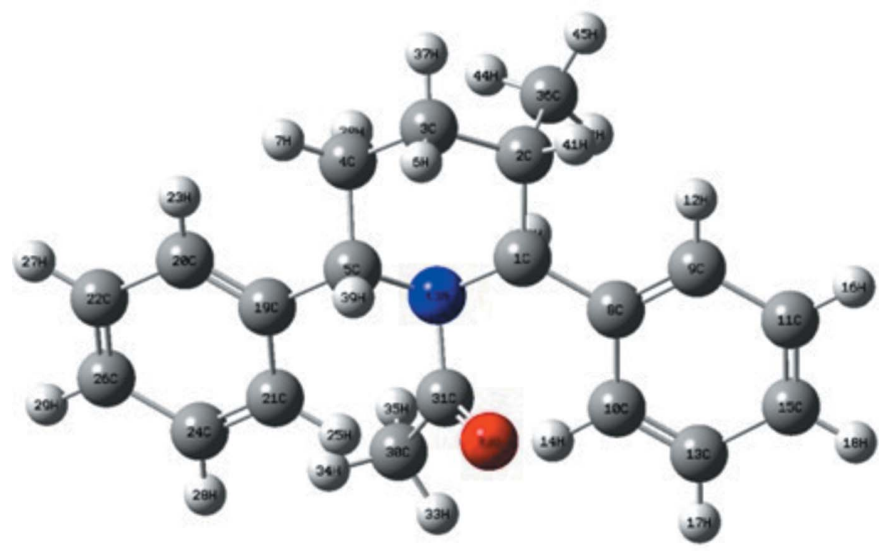

Figure 4

The optimized molecular structure of the title compound.
Table 2

Physico-chemical properties.

\begin{tabular}{lr}
\hline Parameter & \multicolumn{1}{c}{ Value } \\
\hline$E_{\mathrm{HOMO}}(\mathrm{eV})$ & -5.212 \\
$E_{\mathrm{LUMO}}(\mathrm{eV})$ & -2.047 \\
$E_{\mathrm{HOMO}}-E_{\mathrm{LUMO}}$ energy gap $(\mathrm{eV})$ & 3.165 \\
$E_{\mathrm{HOMO}-1}(\mathrm{eV})$ & -5.851 \\
$E_{\mathrm{LUMO}+1}(\mathrm{eV})$ & -2.248 \\
$E_{\mathrm{HOMO}-1}-E_{\mathrm{LUMO}+1}$ energy gap $(\mathrm{eV})$ & 3.603 \\
Ionization potential $I(\mathrm{eV})$ & 5.212 \\
Electron affinity $(A)$ & 2.047 \\
Electrophilicity Index $(\omega)$ & 4.163 \\
Chemical Potential $(\mu)$ & 3.629 \\
Electro negativity $(\chi)$ & -3.630 \\
Hardness $(\eta)$ & 1.583 \\
Softness $(\sigma)$ & 0.316 \\
\hline
\end{tabular}

$\left.E_{\mathrm{HOMO}}-E_{\mathrm{LUMO}}\right)$ of the molecule is $3.165 \mathrm{eV}$ and the calculated frontier molecular orbital energies, $E_{\mathrm{HOMO}}$ and $E_{\mathrm{LUMO}}$, are -5.212 and $-2.047 \mathrm{eV}$, respectively. The title compound has a small frontier orbital gap, hence the molecule has high chemical reactivity and low kinetic stability. The electron affinity $(A)$ and ionization potential $(I)$ of the molecule were calculated using the DFT/B3LYP/6- 311++G(d,p) basis set. The values of the hardness $(\eta)$, softness $(\sigma)$, electronegativity $(\chi)$ and electrophilicity index $(\omega)$ for the title compound are given in Table 2.

\section{Hirshfeld surface analysis}

In order to visualize the intermolecular interactions in the crystal of the title compound, a Hirshfeld surface (HS) analysis (Spackman \& Jayatilaka, 2009) was carried out and the associated two-dimensional fingerprint plots (McKinnon et al., 2007) were generated using CrystalExplorer17 (Turner et al., 2017). The Hirshfeld surface mapped over $d_{\text {norm }}$ using a standard surface resolution with a fixed colour scale of -0.2 (red) to 1.3 (blue) a.u. is shown in Fig. 6a. The shorter and
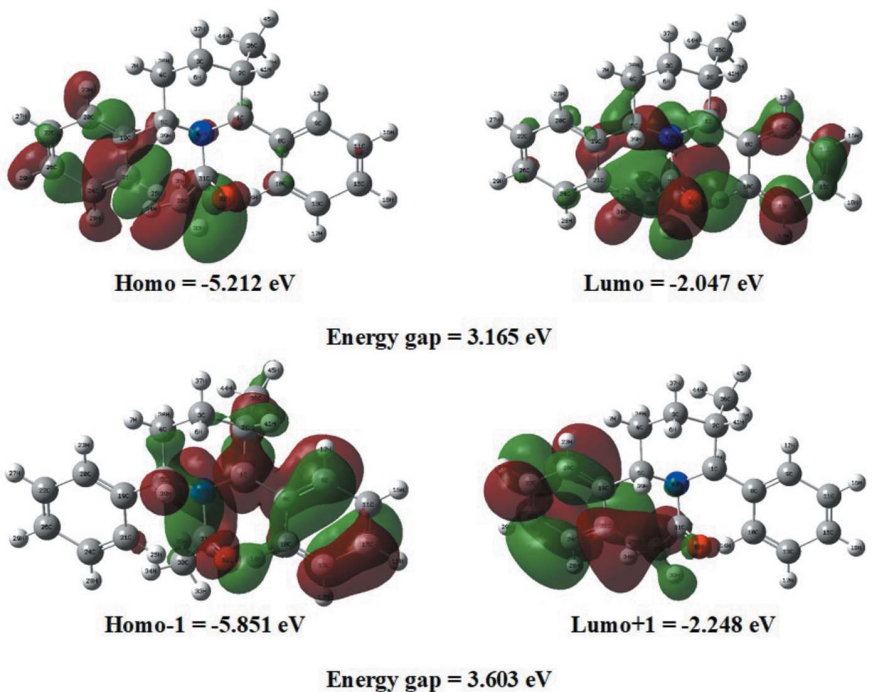

Figure 5

The frontier molecular orbitals (FMOs) of the title compound. 

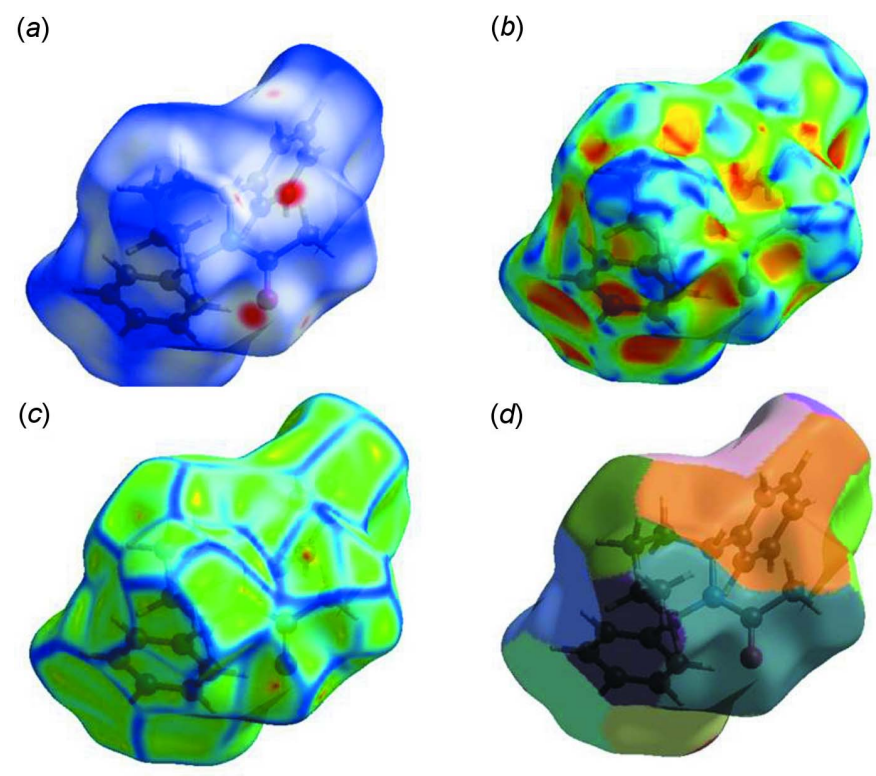

Figure 6

Hirshfeld surfaces mapped over $(a) d_{\text {norm }},(b)$ shape-index, $(c)$ curvedness and $(d)$ fragment patches.

longer contacts are indicated as red and blue spots, respectively, on the Hirshfeld surfaces, and contacts with distances approximately equal to the sum of the van der Waals radii are represented as white spots. The most important red spots on the $d_{\text {norm }}$ surface represent $\mathrm{C}-\mathrm{H} \cdots \mathrm{O}$ interactions.

The HS mapped over curvedness and shape-index, introduced by Koendrink (Koenderink, 1990; Koenderink \& van
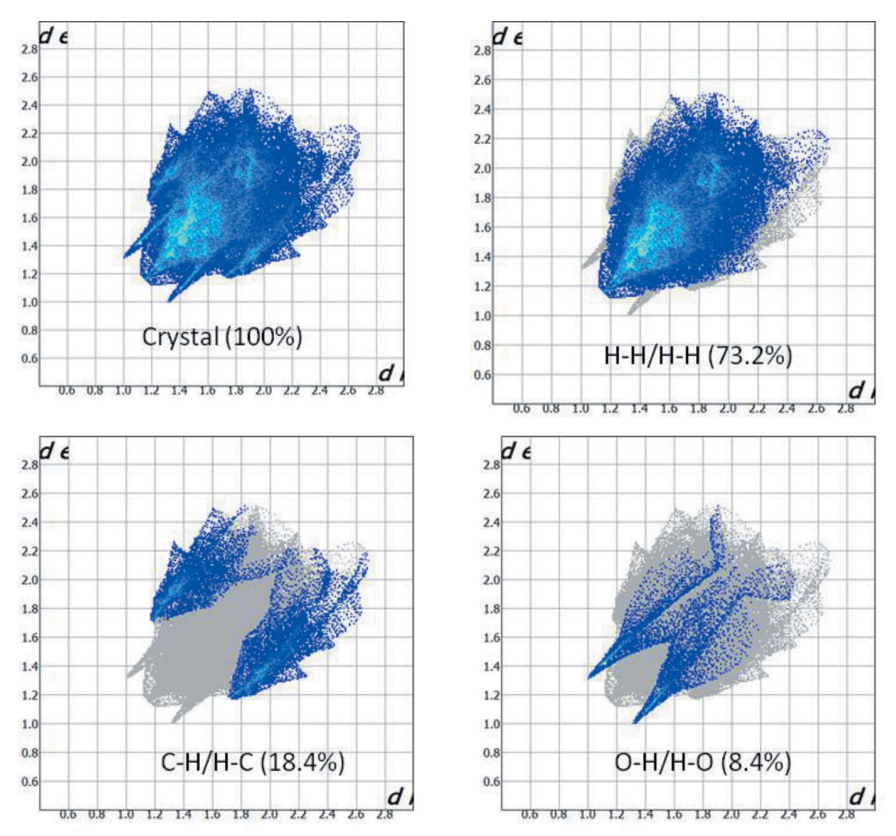

Figure 7

Two-dimensional fingerprint plot for the title compound showing the contributions of individual types of interactions (all intermolecular contacts, $\mathrm{H} \cdots \mathrm{H}$ contacts, $\mathrm{C} \cdots \mathrm{H} / \mathrm{H} \cdots \mathrm{C}$ contacts and $\mathrm{O} \cdots \mathrm{H} / \mathrm{H} \cdots \mathrm{O}$ contacts).
Doorn, 1992), give further chemical insight into molecular packing. A surface with low curvedness designates a flat region and may be indicative of $\pi-\pi$ stacking in the crystal. A surface with high curvedness is highlighted as dark blue edges, and is indicative of the absence of $\pi-\pi$ stacking (Fig. 6). The nearest neighbour coordination environment of a molecule is identified from the colour patches on the Hirshfeld surface, depending on their closeness to adjacent molecules (Mohamooda Sumaya et al., 2017).

The two-dimensional fingerprint plots of $\left(d_{\mathrm{i}}, d_{\mathrm{e}}\right)$ points of all the contacts contributing to the Hirshfeld surface analysis in normal mode for all the atoms are shown in Fig. 7. The most important intermolecular interactions are $\mathrm{H} \cdot \mathrm{H}$ contacts, contributing $73.2 \%$ to the overall crystal packing. Other interactions and their respective contributions are $\mathrm{C} \cdots \mathrm{H}$ / $\mathrm{H} \cdots \mathrm{C}(18.4 \%)$ and $\mathrm{O} \cdots \mathrm{H} / \mathrm{H} \cdots \mathrm{O}(8.4 \%)$, respectively.

The Hirshfeld surface analysis confirms the importance of $\mathrm{H}$-atom contacts in establishing the packing. The large number of $\mathrm{H} \cdots \mathrm{H}$ and $\mathrm{C} \cdots \mathrm{H} / \mathrm{H} \cdots \mathrm{C}$ interactions suggest that van der Waals interactions and hydrogen bonding play the major roles in the crystal packing (Hathwar et al., 2015).

\section{Database survey}

A search of the Cambridge Structural Database (CSD, version 5.39, update August 2018; Groom et al., 2016) for the 3-methyl2,6-diphenylpiperidine skeleton yielded two hits, methyl 4-oxo-r-2,c-6-diphenylpiperidine-3-carboxylate (BIHZEY; Sampath et al., 2004) and r-2,c-6-diphenylpiperidine (NIKYEN; Maheshwaran et al., 2013). The piperidine ring has a boat-shaped conformation in both compounds, as in the title compound. The benzene ring and the mean plane of the piperidine ring are inclined to each other by dihedral angles ranging from 19.95 to $29.16^{\circ}$, compared to $22.05(6)^{\circ}$ in the title compound.

\section{Synthesis and crystallization}

The compound $t$-3-methyl-r-2,c-6-diphenylpiperidin-4-one was reduced to the corresponding piperidine using the WolfKishner reduction (Ravindran \& Jeyaraman, 1992). The piperidine-4-one $(10 \mathrm{mmol})$ was treated with diethylene glycol (40 $\mathrm{ml})$, hydrazine hydrate $(10 \mathrm{mmol})$ and $\mathrm{KOH}$ pellets (10 mmol) to give $t$-3-methyl- $r$-2,c-6-diphenylpiperidine. $N$ Acetyl piperidine was synthesized by the acetylation of the above piperidine. To $t$-3-methyl- $r$-2,c-6-diphenylpiperidine $(5 \mathrm{mmol})$ dissolved in benzene $(50 \mathrm{ml})$ were added triethylamine $(20 \mathrm{mmol})$ and acetyl chloride $(20 \mathrm{mmol})$ to give $N$ acetyl-t-3-methyl-r-2,c-6-diphenylpiperidine, which was crystallized by slow evaporation from a benzene and petroleum ether solution.

\section{Refinement}

Crystal data, data collection and structure refinement details are summarized in Table 3 . $\mathrm{H}$ atoms were positioned geometrically $(\mathrm{N}-\mathrm{H}=0.88-0.90 \AA$ and $\mathrm{C}-\mathrm{H}=0.93-0.98 \AA)$ and 
Table 3

Experimental details.

\begin{tabular}{|c|c|}
\hline \multicolumn{2}{|l|}{ Crystal data } \\
\hline Chemical formula & $\mathrm{C}_{20} \mathrm{H}_{23} \mathrm{NO}$ \\
\hline$M_{\mathrm{r}}$ & 293.39 \\
\hline Crystal system, space group & Monoclinic, $P 2_{1}$ \\
\hline Temperature (K) & 296 \\
\hline$a, b, c(\AA)$ & $8.3063(4), 7.5842(4), 13.8410(7)$ \\
\hline$\beta\left(^{\circ}\right)$ & $104.174(2)$ \\
\hline$V\left(\AA^{3}\right)$ & $845.39(7)$ \\
\hline$Z$ & 2 \\
\hline Radiation type & Мо $K \alpha$ \\
\hline$\mu\left(\mathrm{mm}^{-1}\right)$ & 0.07 \\
\hline Crystal size $(\mathrm{mm})$ & $0.30 \times 0.25 \times 0.25$ \\
\hline \multicolumn{2}{|l|}{ Data collection } \\
\hline Diffractometer & Bruker SMART APEXII CCD \\
\hline Absorption correction & $\begin{array}{l}\text { Multi-scan (SADABS; Bruker, } \\
\text { 2016) }\end{array}$ \\
\hline$T_{\min }, T_{\max }$ & $0.697,0.745$ \\
\hline $\begin{array}{l}\text { No. of measured, independent and } \\
\text { observed }[I>2 \sigma(I)] \text { reflections }\end{array}$ & $15383,3447,2821$ \\
\hline$R_{\text {int }}$ & 0.024 \\
\hline$(\sin \theta / \lambda)_{\max }\left(\AA^{-1}\right)$ & 0.626 \\
\hline \multicolumn{2}{|l|}{ Refinement } \\
\hline$R\left[F^{2}>2 \sigma\left(F^{2}\right)\right], w R\left(F^{2}\right), S$ & $0.037,0.101,1.03$ \\
\hline No. of reflections & 3447 \\
\hline No. of parameters & 201 \\
\hline No. of restraints & 1 \\
\hline $\mathrm{H}$-atom treatment & $\mathrm{H}$-atom parameters constrained \\
\hline$\Delta \rho_{\max }, \Delta \rho_{\min }\left(\mathrm{e} \AA^{-3}\right)$ & $0.22,-0.13$ \\
\hline Absolute structure & $\begin{array}{l}\text { Flack } x \text { determined using } 1136 \\
\quad \text { quotients }\left[\left(I^{+}\right)-\left(I^{-}\right)\right] /\left[\left(I^{+}\right)+\left(I^{-}\right)\right] \\
\quad \text { (Parsons } \text { et al., 2013) }\end{array}$ \\
\hline Absolute structure parameter & $0.0(5)$ \\
\hline
\end{tabular}

Computer programs: APEX2 and SAINT (Bruker, 2016), SHELXS97 and SHELXL97 (Sheldrick, 2008), SHELXL2018/3 (Sheldrick, 2015), ORTEP-3 for Windows (Farrugia, 2012) and PLATON (Spek, 2020).

allowed to ride on their parent atoms, with $U_{\text {iso }}(\mathrm{H})=$ $1.5 \mathrm{U}_{\mathrm{eq}}(\mathrm{C})$ for methyl $\mathrm{H}$ and $1.2 U_{\mathrm{eq}}(\mathrm{C})$ for other $\mathrm{H}$ atoms.

\section{Acknowledgements}

The authors thank the SAIF, IIT Madras, India, for the data collection.

\section{Funding information}

KR thanks the UGC, New Delhi, for financial assistance in the form of a Minor Research Project.

\section{References}

Aridoss, G., Parthiban, P., Ramachandran, R., Prakash, M., Kabilan, S. \& Jeong, Y. T. (2009). Eur. J. Med. Chem. 44, 577-592.

Beddoes, R. L., Dalton, L., Joule, T. A., Mills, O. S., Street, J. D. \& Watt, C. I. F. (1986). J. Chem. Soc. Perkin Trans. 2, pp. 787-797.

Bernstein, J., Davis, R. E., Shimoni, L. \& Chang, N. L. (1995). Angew. Chem. Int. Ed. Engl. 34, 1555-1573.
Bruker (2016). APEX3, SAINT and SADABS. Bruker AXS Inc., Madison, Wisconsin, USA.

Cremer, D. \& Pople, J. A. (1975). J. Am. Chem. Soc. 97, 1354-1358. Farrugia, L. J. (2012). J. Appl. Cryst. 45, 849-854.

Frisch, M. J., Trucks, G. W., Schlegel, H. B., Scuseria, G. E., Robb, M. A., Cheeseman, J. R., Scalmani, G., Barone, V., Mennucci, B., Petersson, G. A., Nakatsuji, H., Caricato, M., Li, X., Hratchian, H. P., Izmaylov, A. F., Bloino, J., Zheng, G., Sonnenberg, J. L., Hada, M., Ehara, M., Toyota, K., Fukuda, R., Hasegawa, J., Ishida, M., Nakajima, T., Honda, Y., Kitao, O., Nakai, H., Vreven, T., Montgomery, J. A. Jr, Peralta, J. E., Ogliaro, F., Bearpark, M., Heyd, J. J., Brothers, E., Kudin, K. N., Staroverov, V. N., Kobayashi, R., Normand, J., Raghavachari, K., Rendell, A., Burant, J. C., Iyengar, S. S., Tomasi, J., Cossi, M., Rega, N., Millam, J. M., Klene, M., Knox, J. E., Cross, J. B., Bakken, V., Adamo, C., Jaramillo, J., Gomperts, R., Stratmann, R. E., Yazyev, O., Austin, A. J., Cammi, R., Pomelli, C., Ochterski, J. W., Martin, R. L., Morokuma, K., Zakrzewski, V. G., Voth, G. A., Salvador, P., Dannenberg, J. J., Dapprich, S., Daniels, A. D., Farkas, Ö., Foresman, J. B., Ortiz, J. V., Cioslowski, J. \& Fox, D. J. (2009). GAUSSIAN09. Gaussian Inc., Wallingford, CT, USA.

Groom, C. R., Bruno, I. J., Lightfoot, M. P. \& Ward, S. C. (2016). Acta Cryst. B72, 171-179.

Hathwar, V. R., Sist, M., Jørgensen, M. R. V., Mamakhel, A. H., Wang, X., Hoffmann, C. M., Sugimoto, K., Overgaard, J. \& Iversen, B. B. (2015). IUCrJ, 2, 563-574.

Katritzky, A. (2010). Adv. Heterocycl. Chem. pp. 42-89.

Koenderink, J. J. (1990). Solid Shape. Cambridge MA: MIT Press.

Koenderink, J. J. \& van Doorn, A. J. (1992). Image Vis. Comput. 10, 557-564.

Maheshwaran, V., Abdul Basheer, S., Akila, A., Ponnuswamy, S. \& Ponnuswamy, M. N. (2013). Acta Cryst. E69, o1371.

McKinnon, J. J., Jayatilaka, D. \& Spackman, M. A. (2007). Chem. Commun. pp. 3814-3816.

Mobio, I. G., Soldatenkov, A. T., Federov, V. O., Ageev, E. A., Sargeeva, N. D., Lin, S., Stashenko, E. E., Prostakov, N. S. \& \&Andreeva, E. I. (1989). Khim. Farm. Zh. 23, 421-427.

Mohamooda Sumaya, U., Reuben Jonathan, D., Era, D. T., Gomathi, S. \& Usha, G. (2017). IUCrData, 2, x170813.

Nardelli, M. (1983). Acta Cryst. C39, 1141-1142.

Parsons, S., Flack, H. D. \& Wagner, T. (2013). Acta Cryst. B69, 249259.

Ravindran, T. \& Jeyaraman, R. (1992). Indian J. Chem. B31, 677-682.

Roques, R., Declercq, J. P., Germain, G., Graffin, P., Kamenka, J. M. \& Geneste, P. (1981). Acta Cryst. B37, 712-714.

Sampath, N., Aravindhan, S., Ponnuswamy, M. N. \& Nethaji, M. (2004). Acta Cryst. E60, o2105-o2106.

Sekar, K., Parthasarathy, S. \& Radhakrishnan, T. R. (1990). Acta Cryst. C46, 1338-1340.

Sheldrick, G. M. (2008). Acta Cryst. A64, 112-122.

Sheldrick, G. M. (2015). Acta Cryst. C71, 3-8.

Spackman, M. A. \& Jayatilaka, D. (2009). CrystEngComm, 11, 19-32. Spek, A. L. (2020). Acta Cryst. E76, 1-11.

Turner, M. J., MacKinnon, J. J., Wolff, S. K., Grimwood, D. J., Spackman, P. R., Jayatilaka, D. \& Spackman, M. A. (2017). Crystal Explorer 17.5. University of Western Australia.

Vinaya, K., Kavitha, R., Ananda Kumar, C. S., Benaka Prasad, S. B., Chandrappa, S., Deepak, S. A., NanjundaSwamy, S., Umesha, S. \& Rangappa, K. S. (2009). Arch. Pharm. Res. 32, 1, 33-41. 


\section{supporting information}

Acta Cryst. (2022). E78, 179-183 [https://doi.org/10.1107/S2056989022000275]

Crystal structure, DFT and Hirshfeld surface analysis of $\mathrm{N}$-acetyl-t-3-methyl$r$-2,c-6-diphenylpiperidine

\section{P. Periyannan, M. Beemarao, K . Karthik, S. Ponnuswamy and K. Ravichandran}

Computing details

Data collection: APEX2 (Bruker, 2016); cell refinement: SAINT (Bruker, 2016); data reduction: SAINT (Bruker, 2016); program(s) used to solve structure: SHELXS97 (Sheldrick, 2008); program(s) used to refine structure: SHELXL-2018/3 (Sheldrick, 2015); molecular graphics: ORTEP-3 for Windows (Farrugia, 2012); software used to prepare material for publication: SHELXL97 and PLATON (Spek, 2020).

1-(3-Methyl-2,6-diphenylpiperidin-1-yl)ethanone

Crystal data

$\mathrm{C}_{20} \mathrm{H}_{23} \mathrm{NO}$

$M_{r}=293.39$

Monoclinic, $P 2_{1}$

$a=8.3063$ (4) $\AA$

$b=7.5842(4) \AA$

$c=13.8410(7) \AA$

$\beta=104.174(2)^{\circ}$

$V=845.39(7) \AA^{3}$

$Z=2$

\section{Data collection}

Bruker SMART APEXII CCD diffractometer

Radiation source: fine-focus sealed tube $\omega$ and $\varphi$ scans

Absorption correction: multi-scan

(SADABS; Bruker, 2016)

$T_{\min }=0.697, T_{\max }=0.745$

15383 measured reflections

\section{Refinement}

Refinement on $F^{2}$

Least-squares matrix: full

$R\left[F^{2}>2 \sigma\left(F^{2}\right)\right]=0.037$

$w R\left(F^{2}\right)=0.101$

$S=1.03$

3447 reflections

201 parameters

1 restraint

Primary atom site location: difference Fourier

map
$F(000)=316$

$D_{\mathrm{x}}=1.153 \mathrm{Mg} \mathrm{m}^{-3}$

Mo $K \alpha$ radiation, $\lambda=0.71073 \AA$

Cell parameters from 2821 reflections

$\theta=2.6-26.4^{\circ}$

$\mu=0.07 \mathrm{~mm}^{-1}$

$T=296 \mathrm{~K}$

Block, white

$0.30 \times 0.25 \times 0.25 \mathrm{~mm}$

3447 independent reflections

2821 reflections with $I>2 \sigma(I)$

$R_{\text {int }}=0.024$

$\theta_{\text {max }}=26.4^{\circ}, \theta_{\min }=2.6^{\circ}$

$h=-10 \rightarrow 9$

$k=-9 \rightarrow 9$

$l=-17 \rightarrow 17$

Hydrogen site location: inferred from neighbouring sites

$\mathrm{H}$-atom parameters constrained

$w=1 /\left[\sigma^{2}\left(F_{\mathrm{o}}^{2}\right)+(0.0534 P)^{2}+0.0626 P\right]$

where $P=\left(F_{\mathrm{o}}^{2}+2 F_{\mathrm{c}}^{2}\right) / 3$

$(\Delta / \sigma)_{\max }<0.001$

$\Delta \rho_{\max }=0.22 \mathrm{e} \AA^{-3}$

$\Delta \rho_{\min }=-0.13$ e $\AA^{-3}$ 
Absolute structure: Flack $x$ determined using

1136 quotients $\left[\left(I^{+}\right)-(I)\right] /\left[\left(I^{+}\right)+(I)\right]$ (Parsons et al., 2013)

Absolute structure parameter: 0.0 (5)

Special details

Geometry. All esds (except the esd in the dihedral angle between two 1.s. planes) are estimated using the full covariance matrix. The cell esds are taken into account individually in the estimation of esds in distances, angles and torsion angles; correlations between esds in cell parameters are only used when they are defined by crystal symmetry. An approximate (isotropic) treatment of cell esds is used for estimating esds involving l.s. planes.

Fractional atomic coordinates and isotropic or equivalent isotropic displacement parameters $\left(\AA^{2}\right)$

\begin{tabular}{|c|c|c|c|c|}
\hline & $x$ & $y$ & $z$ & $U_{\text {iso }} * / U_{\text {eq }}$ \\
\hline $\mathrm{C} 2$ & $0.3371(3)$ & $0.2057(3)$ & $0.31568(16)$ & $0.0457(5)$ \\
\hline $\mathrm{H} 2$ & 0.377220 & 0.270737 & 0.378151 & $0.055^{*}$ \\
\hline $\mathrm{C} 3$ & $0.1580(3)$ & $0.2626(4)$ & $0.2699(2)$ & $0.0565(6)$ \\
\hline $\mathrm{H} 3 \mathrm{~A}$ & 0.155366 & 0.388579 & 0.257504 & $0.068 *$ \\
\hline H3B & 0.117951 & 0.203547 & 0.206358 & $0.068 *$ \\
\hline $\mathrm{C} 4$ & $0.0435(3)$ & $0.2199(4)$ & $0.3367(2)$ & $0.0679(7)$ \\
\hline $\mathrm{H} 4 \mathrm{~A}$ & -0.070712 & 0.231457 & 0.298638 & $0.081 *$ \\
\hline H4B & 0.061680 & 0.304992 & 0.390657 & $0.081 *$ \\
\hline $\mathrm{C} 5$ & $0.0700(3)$ & $0.0344(4)$ & $0.38074(17)$ & $0.0567(6)$ \\
\hline H5 & -0.038621 & -0.023553 & 0.368498 & $0.068 *$ \\
\hline C6 & $0.1821(3)$ & $-0.0774(3)$ & $0.33117(17)$ & $0.0481(5)$ \\
\hline H6 & 0.209751 & -0.182736 & 0.372930 & $0.058 *$ \\
\hline $\mathrm{C} 7$ & 0.1060 & $-0.1457(3)$ & $0.22655(17)$ & $0.0502(5)$ \\
\hline $\mathrm{C} 8$ & -0.0600 & $-0.1247(4)$ & $0.1781(2)$ & $0.0659(7)$ \\
\hline $\mathrm{H} 8$ & -0.129032 & -0.058009 & 0.207568 & $0.079 *$ \\
\hline C9 & $-0.1240(5)$ & $-0.2032(5)$ & $0.0855(2)$ & $0.0812(10)$ \\
\hline H9 & -0.235412 & -0.188306 & 0.053477 & $0.097^{*}$ \\
\hline $\mathrm{C} 10$ & $-0.0235(6)$ & $-0.3020(5)$ & $0.0415(2)$ & $0.0890(11)$ \\
\hline $\mathrm{H} 10$ & -0.066947 & -0.354500 & -0.020068 & $0.107^{*}$ \\
\hline $\mathrm{C} 11$ & $0.1404(5)$ & $-0.3236(4)$ & $0.0881(2)$ & $0.0824(10)$ \\
\hline H11 & 0.208456 & -0.390605 & 0.058133 & $0.099 *$ \\
\hline $\mathrm{C} 12$ & $0.2053(4)$ & $-0.2460(4)$ & $0.1796(2)$ & $0.0637(7)$ \\
\hline H12 & 0.317158 & -0.260974 & 0.210498 & $0.076^{*}$ \\
\hline $\mathrm{C} 13$ & $0.4446(3)$ & 0.2557 & $0.24606(16)$ & $0.0471(5)$ \\
\hline $\mathrm{C} 14$ & $0.4544(3)$ & 0.1539 (4) & 0.16487 (19) & $0.0584(6)$ \\
\hline H14 & 0.395691 & 0.048462 & 0.152879 & $0.070 *$ \\
\hline $\mathrm{C} 15$ & $0.5502(4)$ & $0.2063(5)$ & $0.1012(2)$ & $0.0715(8)$ \\
\hline H15 & 0.555322 & 0.136101 & 0.047006 & $0.086^{*}$ \\
\hline $\mathrm{C} 16$ & $0.6372(4)$ & $0.3607(5)$ & $0.1175(2)$ & $0.0783(9)$ \\
\hline H16 & 0.701865 & 0.395466 & 0.074701 & $0.094 *$ \\
\hline $\mathrm{C} 17$ & 0.6289 (4) & $0.4638(4)$ & $0.1971(3)$ & $0.0775(9)$ \\
\hline $\mathrm{H} 17$ & 0.688101 & 0.568970 & 0.208368 & $0.093 *$ \\
\hline $\mathrm{C} 18$ & $0.5326(3)$ & $0.4126(4)$ & $0.2611(2)$ & $0.0632(7)$ \\
\hline H18 & 0.526974 & 0.484386 & 0.314655 & $0.076^{*}$ \\
\hline C19 & $0.4825(3)$ & $-0.0674(3)$ & $0.39016(16)$ & $0.0476(5)$ \\
\hline
\end{tabular}




\begin{tabular}{lllll}
$\mathrm{C} 20$ & $0.6396(3)$ & $0.0380(4)$ & $0.41982(19)$ & $0.0617(7)$ \\
$\mathrm{H} 20 \mathrm{~A}$ & 0.724197 & -0.031394 & 0.462980 & $0.093^{*}$ \\
$\mathrm{H} 20 \mathrm{~B}$ & 0.619811 & 0.142671 & 0.454141 & $0.093^{*}$ \\
$\mathrm{H} 20 \mathrm{C}$ & 0.675456 & 0.070131 & 0.361322 & $0.093^{*}$ \\
$\mathrm{C} 21$ & $0.1416(4)$ & $0.0413(5)$ & $0.4921(2)$ & $0.0733(8)$ \\
$\mathrm{H} 21 \mathrm{~A}$ & 0.155370 & -0.076398 & 0.518301 & $0.110^{*}$ \\
$\mathrm{H} 21 \mathrm{~B}$ & 0.067488 & 0.105297 & 0.522752 & $0.110^{*}$ \\
$\mathrm{H} 21 \mathrm{C}$ & 0.247396 & 0.099569 & 0.506051 & $0.110^{*}$ \\
$\mathrm{~N} 1$ & $0.3411(2)$ & $0.0152(2)$ & $0.33954(13)$ & $0.0433(4)$ \\
$\mathrm{O} 1$ & $0.4827(2)$ & $-0.2245(3)$ & $0.41220(16)$ & $0.0707(5)$ \\
\hline
\end{tabular}

Atomic displacement parameters $\left(\AA^{2}\right)$

\begin{tabular}{lllllll}
\hline & $U^{11}$ & $U^{22}$ & $U^{33}$ & $U^{12}$ & $U^{13}$ & $U^{23}$ \\
\hline C2 & $0.0422(11)$ & $0.0485(13)$ & $0.0457(11)$ & $0.0023(10)$ & $0.0097(9)$ & $-0.0057(10)$ \\
C3 & $0.0478(13)$ & $0.0552(14)$ & $0.0642(14)$ & $0.0106(11)$ & $0.0097(11)$ & $0.0003(12)$ \\
C4 & $0.0484(14)$ & $0.0814(19)$ & $0.0771(17)$ & $0.0154(14)$ & $0.0216(13)$ & $-0.0054(15)$ \\
C5 & $0.0388(12)$ & $0.0813(18)$ & $0.0536(13)$ & $-0.0051(12)$ & $0.0180(10)$ & $-0.0059(13)$ \\
C6 & $0.0379(11)$ & $0.0585(13)$ & $0.0484(11)$ & $-0.0038(10)$ & $0.0117(9)$ & $0.0029(11)$ \\
C7 & $0.0493(13)$ & $0.0504(12)$ & $0.0509(12)$ & $-0.0083(11)$ & $0.0123(10)$ & $0.0014(11)$ \\
C8 & $0.0556(15)$ & $0.0738(19)$ & $0.0623(15)$ & $-0.0092(14)$ & $0.0029(12)$ & $-0.0028(14)$ \\
C9 & $0.080(2)$ & $0.085(2)$ & $0.0654(17)$ & $-0.0241(17)$ & $-0.0076(16)$ & $0.0078(16)$ \\
C10 & $0.122(3)$ & $0.086(2)$ & $0.0541(16)$ & $-0.035(2)$ & $0.0132(19)$ & $-0.0097(16)$ \\
C11 & $0.109(3)$ & $0.071(2)$ & $0.0726(19)$ & $-0.0196(18)$ & $0.0327(19)$ & $-0.0186(16)$ \\
C12 & $0.0700(17)$ & $0.0594(15)$ & $0.0644(15)$ & $-0.0064(13)$ & $0.0217(13)$ & $-0.0078(13)$ \\
C13 & $0.0420(12)$ & $0.0468(12)$ & $0.0502(12)$ & $0.0015(10)$ & $0.0068(9)$ & $0.0034(10)$ \\
C14 & $0.0599(14)$ & $0.0624(15)$ & $0.0547(14)$ & $-0.0057(12)$ & $0.0173(12)$ & $-0.0007(11)$ \\
C15 & $0.0708(17)$ & $0.088(2)$ & $0.0616(15)$ & $0.0017(16)$ & $0.0277(14)$ & $0.0080(15)$ \\
C16 & $0.0658(18)$ & $0.092(2)$ & $0.083(2)$ & $-0.0003(17)$ & $0.0287(15)$ & $0.0310(19)$ \\
C17 & $0.0677(17)$ & $0.0639(18)$ & $0.102(2)$ & $-0.0122(14)$ & $0.0221(17)$ & $0.0219(17)$ \\
C18 & $0.0586(15)$ & $0.0526(14)$ & $0.0755(16)$ & $-0.0018(12)$ & $0.0110(13)$ & $0.0047(13)$ \\
C19 & $0.0417(12)$ & $0.0580(14)$ & $0.0444(11)$ & $0.0082(11)$ & $0.0129(9)$ & $0.0067(10)$ \\
C20 & $0.0361(11)$ & $0.0821(18)$ & $0.0650(14)$ & $0.0069(12)$ & $0.0085(10)$ & $0.0051(14)$ \\
C21 & $0.0709(18)$ & $0.091(2)$ & $0.0638(15)$ & $-0.0030(16)$ & $0.0278(13)$ & $0.0006(16)$ \\
N1 & $0.0335(9)$ & $0.0520(10)$ & $0.0445(9)$ & $0.0014(8)$ & $0.0100(7)$ & $0.0023(8)$ \\
O1 & $0.0595(12)$ & $0.0657(12)$ & $0.0843(13)$ & $0.0117(9)$ & $0.0125(10)$ & $0.0206(10)$ \\
& & & & & & \\
\hline
\end{tabular}

Geometric parameters $\left(A,{ }^{o}\right)$

\begin{tabular}{llll}
\hline $\mathrm{C} 2-\mathrm{N} 1$ & $1.481(3)$ & $\mathrm{C} 11-\mathrm{C} 12$ & $1.381(4)$ \\
$\mathrm{C} 2-\mathrm{C} 13$ & $1.513(3)$ & $\mathrm{C} 11-\mathrm{H} 11$ & 0.9300 \\
$\mathrm{C} 2-\mathrm{C} 3$ & $1.530(3)$ & $\mathrm{C} 12-\mathrm{H} 12$ & 0.9300 \\
$\mathrm{C} 2-\mathrm{H} 2$ & 0.9800 & $\mathrm{C} 13-\mathrm{C} 14$ & $1.382(4)$ \\
$\mathrm{C} 3-\mathrm{C} 4$ & $1.515(4)$ & $\mathrm{C} 13-\mathrm{C} 18$ & $1.386(4)$ \\
$\mathrm{C} 3-\mathrm{H} 3 \mathrm{~A}$ & 0.9700 & $\mathrm{C} 14-\mathrm{C} 15$ & $1.382(4)$ \\
$\mathrm{C} 3-\mathrm{H} 3 \mathrm{~B}$ & 0.9700 & $\mathrm{C} 14-\mathrm{H} 14$ & 0.9300 \\
$\mathrm{C} 4-\mathrm{C} 5$ & $1.528(4)$ & $\mathrm{C} 15-\mathrm{C} 16$ & $1.366(5)$ \\
$\mathrm{C} 4-\mathrm{H} 4 \mathrm{~A}$ & 0.9700 & $\mathrm{C} 15-\mathrm{H} 15$ & 0.9300
\end{tabular}




\begin{tabular}{|c|c|c|c|}
\hline $\mathrm{C} 4-\mathrm{H} 4 \mathrm{~B}$ & 0.9700 & $\mathrm{C} 16-\mathrm{C} 17$ & $1.366(5)$ \\
\hline $\mathrm{C} 5-\mathrm{C} 21$ & $1.511(4)$ & $\mathrm{C} 16-\mathrm{H} 16$ & 0.9300 \\
\hline $\mathrm{C} 5-\mathrm{C} 6$ & $1.539(3)$ & $\mathrm{C} 17-\mathrm{C} 18$ & $1.386(4)$ \\
\hline $\mathrm{C} 5-\mathrm{H} 5$ & 0.9800 & $\mathrm{C} 17-\mathrm{H} 17$ & 0.9300 \\
\hline $\mathrm{C} 6-\mathrm{N} 1$ & $1.475(3)$ & $\mathrm{C} 18-\mathrm{H} 18$ & 0.9300 \\
\hline $\mathrm{C} 6-\mathrm{C} 7$ & $1.522(3)$ & $\mathrm{C} 19-\mathrm{O} 1$ & $1.230(3)$ \\
\hline $\mathrm{C} 6-\mathrm{H} 6$ & 0.9800 & $\mathrm{C} 19-\mathrm{N} 1$ & $1.364(3)$ \\
\hline $\mathrm{C} 7-\mathrm{C} 8$ & $1.387(3)$ & $\mathrm{C} 19-\mathrm{C} 20$ & $1.499(4)$ \\
\hline $\mathrm{C} 7-\mathrm{C} 12$ & $1.394(4)$ & $\mathrm{C} 20-\mathrm{H} 20 \mathrm{~A}$ & 0.9600 \\
\hline $\mathrm{C} 8-\mathrm{C} 9$ & $1.395(4)$ & $\mathrm{C} 20-\mathrm{H} 20 \mathrm{~B}$ & 0.9600 \\
\hline $\mathrm{C} 8-\mathrm{H} 8$ & 0.9300 & $\mathrm{C} 20-\mathrm{H} 20 \mathrm{C}$ & 0.9600 \\
\hline $\mathrm{C} 9-\mathrm{C} 10$ & $1.371(6)$ & $\mathrm{C} 21-\mathrm{H} 21 \mathrm{~A}$ & 0.9600 \\
\hline C9-H9 & 0.9300 & $\mathrm{C} 21-\mathrm{H} 21 \mathrm{~B}$ & 0.9600 \\
\hline $\mathrm{C} 10-\mathrm{C} 11$ & $1.366(5)$ & $\mathrm{C} 21-\mathrm{H} 21 \mathrm{C}$ & 0.9600 \\
\hline $\mathrm{C} 10-\mathrm{H} 10$ & 0.9300 & & \\
\hline $\mathrm{N} 1-\mathrm{C} 2-\mathrm{C} 13$ & $113.69(18)$ & $\mathrm{C} 10-\mathrm{C} 11-\mathrm{C} 12$ & $120.1(3)$ \\
\hline $\mathrm{N} 1-\mathrm{C} 2-\mathrm{C} 3$ & $109.49(19)$ & $\mathrm{C} 10-\mathrm{C} 11-\mathrm{H} 11$ & 119.9 \\
\hline $\mathrm{C} 13-\mathrm{C} 2-\mathrm{C} 3$ & $109.34(19)$ & $\mathrm{C} 12-\mathrm{C} 11-\mathrm{H} 11$ & 119.9 \\
\hline $\mathrm{N} 1-\mathrm{C} 2-\mathrm{H} 2$ & 108.1 & $\mathrm{C} 11-\mathrm{C} 12-\mathrm{C} 7$ & $121.2(3)$ \\
\hline $\mathrm{C} 13-\mathrm{C} 2-\mathrm{H} 2$ & 108.1 & $\mathrm{C} 11-\mathrm{C} 12-\mathrm{H} 12$ & 119.4 \\
\hline $\mathrm{C} 3-\mathrm{C} 2-\mathrm{H} 2$ & 108.1 & $\mathrm{C} 7-\mathrm{C} 12-\mathrm{H} 12$ & 119.4 \\
\hline $\mathrm{C} 4-\mathrm{C} 3-\mathrm{C} 2$ & $112.3(2)$ & $\mathrm{C} 14-\mathrm{C} 13-\mathrm{C} 18$ & $117.9(2)$ \\
\hline $\mathrm{C} 4-\mathrm{C} 3-\mathrm{H} 3 \mathrm{~A}$ & 109.2 & $\mathrm{C} 14-\mathrm{C} 13-\mathrm{C} 2$ & $122.6(2)$ \\
\hline $\mathrm{C} 2-\mathrm{C} 3-\mathrm{H} 3 \mathrm{~A}$ & 109.2 & $\mathrm{C} 18-\mathrm{C} 13-\mathrm{C} 2$ & $119.4(2)$ \\
\hline $\mathrm{C} 4-\mathrm{C} 3-\mathrm{H} 3 \mathrm{~B}$ & 109.2 & $\mathrm{C} 15-\mathrm{C} 14-\mathrm{C} 13$ & $121.0(3)$ \\
\hline $\mathrm{C} 2-\mathrm{C} 3-\mathrm{H} 3 \mathrm{~B}$ & 109.2 & $\mathrm{C} 15-\mathrm{C} 14-\mathrm{H} 14$ & 119.5 \\
\hline $\mathrm{H} 3 \mathrm{~A}-\mathrm{C} 3-\mathrm{H} 3 \mathrm{~B}$ & 107.9 & $\mathrm{C} 13-\mathrm{C} 14-\mathrm{H} 14$ & 119.5 \\
\hline $\mathrm{C} 3-\mathrm{C} 4-\mathrm{C} 5$ & $112.9(2)$ & $\mathrm{C} 16-\mathrm{C} 15-\mathrm{C} 14$ & $120.4(3)$ \\
\hline $\mathrm{C} 3-\mathrm{C} 4-\mathrm{H} 4 \mathrm{~A}$ & 109.0 & $\mathrm{C} 16-\mathrm{C} 15-\mathrm{H} 15$ & 119.8 \\
\hline $\mathrm{C} 5-\mathrm{C} 4-\mathrm{H} 4 \mathrm{~A}$ & 109.0 & $\mathrm{C} 14-\mathrm{C} 15-\mathrm{H} 15$ & 119.8 \\
\hline $\mathrm{C} 3-\mathrm{C} 4-\mathrm{H} 4 \mathrm{~B}$ & 109.0 & $\mathrm{C} 17-\mathrm{C} 16-\mathrm{C} 15$ & $119.6(3)$ \\
\hline $\mathrm{C} 5-\mathrm{C} 4-\mathrm{H} 4 \mathrm{~B}$ & 109.0 & $\mathrm{C} 17-\mathrm{C} 16-\mathrm{H} 16$ & 120.2 \\
\hline $\mathrm{H} 4 \mathrm{~A}-\mathrm{C} 4-\mathrm{H} 4 \mathrm{~B}$ & 107.8 & $\mathrm{C} 15-\mathrm{C} 16-\mathrm{H} 16$ & 120.2 \\
\hline $\mathrm{C} 21-\mathrm{C} 5-\mathrm{C} 4$ & $110.9(2)$ & $\mathrm{C} 16-\mathrm{C} 17-\mathrm{C} 18$ & $120.4(3)$ \\
\hline $\mathrm{C} 21-\mathrm{C} 5-\mathrm{C} 6$ & $110.1(2)$ & $\mathrm{C} 16-\mathrm{C} 17-\mathrm{H} 17$ & 119.8 \\
\hline $\mathrm{C} 4-\mathrm{C} 5-\mathrm{C} 6$ & $111.90(19)$ & $\mathrm{C} 18-\mathrm{C} 17-\mathrm{H} 17$ & 119.8 \\
\hline $\mathrm{C} 21-\mathrm{C} 5-\mathrm{H} 5$ & 107.9 & $\mathrm{C} 13-\mathrm{C} 18-\mathrm{C} 17$ & $120.7(3)$ \\
\hline $\mathrm{C} 4-\mathrm{C} 5-\mathrm{H} 5$ & 107.9 & $\mathrm{C} 13-\mathrm{C} 18-\mathrm{H} 18$ & 119.7 \\
\hline $\mathrm{C} 6-\mathrm{C} 5-\mathrm{H} 5$ & 107.9 & $\mathrm{C} 17-\mathrm{C} 18-\mathrm{H} 18$ & 119.7 \\
\hline $\mathrm{N} 1-\mathrm{C} 6-\mathrm{C} 7$ & $113.10(17)$ & $\mathrm{O} 1-\mathrm{C} 19-\mathrm{N} 1$ & $121.4(2)$ \\
\hline $\mathrm{N} 1-\mathrm{C} 6-\mathrm{C} 5$ & $109.31(19)$ & $\mathrm{O} 1-\mathrm{C} 19-\mathrm{C} 20$ & $120.0(2)$ \\
\hline $\mathrm{C} 7-\mathrm{C} 6-\mathrm{C} 5$ & $117.12(19)$ & $\mathrm{N} 1-\mathrm{C} 19-\mathrm{C} 20$ & $118.5(2)$ \\
\hline $\mathrm{N} 1-\mathrm{C} 6-\mathrm{H} 6$ & 105.4 & $\mathrm{C} 19-\mathrm{C} 20-\mathrm{H} 20 \mathrm{~A}$ & 109.5 \\
\hline $\mathrm{C} 7-\mathrm{C} 6-\mathrm{H} 6$ & 105.4 & $\mathrm{C} 19-\mathrm{C} 20-\mathrm{H} 20 \mathrm{~B}$ & 109.5 \\
\hline $\mathrm{C} 5-\mathrm{C} 6-\mathrm{H} 6$ & 105.4 & $\mathrm{H} 20 \mathrm{~A}-\mathrm{C} 20-\mathrm{H} 20 \mathrm{~B}$ & 109.5 \\
\hline $\mathrm{C} 8-\mathrm{C} 7-\mathrm{C} 12$ & $118.0(2)$ & $\mathrm{C} 19-\mathrm{C} 20-\mathrm{H} 20 \mathrm{C}$ & 109.5 \\
\hline $\mathrm{C} 8-\mathrm{C} 7-\mathrm{C} 6$ & $123.5(2)$ & $\mathrm{H} 20 \mathrm{~A}-\mathrm{C} 20-\mathrm{H} 20 \mathrm{C}$ & 109.5 \\
\hline
\end{tabular}




\begin{tabular}{|c|c|c|c|}
\hline $\mathrm{C} 12-\mathrm{C} 7-\mathrm{C} 6$ & $118.3(2)$ & $\mathrm{H} 20 \mathrm{~B}-\mathrm{C} 20-\mathrm{H} 20 \mathrm{C}$ & 109.5 \\
\hline $\mathrm{C} 7-\mathrm{C} 8-\mathrm{C} 9$ & $120.3(3)$ & $\mathrm{C} 5-\mathrm{C} 21-\mathrm{H} 21 \mathrm{~A}$ & 109.5 \\
\hline $\mathrm{C} 7-\mathrm{C} 8-\mathrm{H} 8$ & 119.8 & $\mathrm{C} 5-\mathrm{C} 21-\mathrm{H} 21 \mathrm{~B}$ & 109.5 \\
\hline $\mathrm{C} 9-\mathrm{C} 8-\mathrm{H} 8$ & 119.8 & $\mathrm{H} 21 \mathrm{~A}-\mathrm{C} 21-\mathrm{H} 21 \mathrm{~B}$ & 109.5 \\
\hline $\mathrm{C} 10-\mathrm{C} 9-\mathrm{C} 8$ & $120.3(3)$ & $\mathrm{C} 5-\mathrm{C} 21-\mathrm{H} 21 \mathrm{C}$ & 109.5 \\
\hline $\mathrm{C} 10-\mathrm{C} 9-\mathrm{H} 9$ & 119.8 & $\mathrm{H} 21 \mathrm{~A}-\mathrm{C} 21-\mathrm{H} 21 \mathrm{C}$ & 109.5 \\
\hline $\mathrm{C} 8-\mathrm{C} 9-\mathrm{H} 9$ & 119.8 & $\mathrm{H} 21 \mathrm{~B}-\mathrm{C} 21-\mathrm{H} 21 \mathrm{C}$ & 109.5 \\
\hline $\mathrm{C} 11-\mathrm{C} 10-\mathrm{C} 9$ & $120.1(3)$ & $\mathrm{C} 19-\mathrm{N} 1-\mathrm{C} 6$ & $117.61(18)$ \\
\hline $\mathrm{C} 11-\mathrm{C} 10-\mathrm{H} 10$ & 120.0 & $\mathrm{C} 19-\mathrm{N} 1-\mathrm{C} 2$ & $122.16(19)$ \\
\hline $\mathrm{C} 9-\mathrm{C} 10-\mathrm{H} 10$ & 120.0 & $\mathrm{C} 6-\mathrm{N} 1-\mathrm{C} 2$ & $118.46(17)$ \\
\hline $\mathrm{N} 1-\mathrm{C} 2-\mathrm{C} 3-\mathrm{C} 4$ & $56.0(3)$ & $\mathrm{N} 1-\mathrm{C} 2-\mathrm{C} 13-\mathrm{C} 18$ & $-141.6(2)$ \\
\hline $\mathrm{C} 13-\mathrm{C} 2-\mathrm{C} 3-\mathrm{C} 4$ & $-178.8(2)$ & $\mathrm{C} 3-\mathrm{C} 2-\mathrm{C} 13-\mathrm{C} 18$ & $95.7(3)$ \\
\hline $\mathrm{C} 2-\mathrm{C} 3-\mathrm{C} 4-\mathrm{C} 5$ & $-45.0(3)$ & $\mathrm{C} 18-\mathrm{C} 13-\mathrm{C} 14-\mathrm{C} 15$ & $0.6(4)$ \\
\hline $\mathrm{C} 3-\mathrm{C} 4-\mathrm{C} 5-\mathrm{C} 21$ & $112.4(3)$ & $\mathrm{C} 2-\mathrm{C} 13-\mathrm{C} 14-\mathrm{C} 15$ & $178.1(2)$ \\
\hline $\mathrm{C} 3-\mathrm{C} 4-\mathrm{C} 5-\mathrm{C} 6$ & $-11.0(3)$ & $\mathrm{C} 13-\mathrm{C} 14-\mathrm{C} 15-\mathrm{C} 16$ & $0.0(5)$ \\
\hline $\mathrm{C} 21-\mathrm{C} 5-\mathrm{C} 6-\mathrm{N} 1$ & $-68.0(3)$ & $\mathrm{C} 14-\mathrm{C} 15-\mathrm{C} 16-\mathrm{C} 17$ & $-0.2(5)$ \\
\hline $\mathrm{C} 4-\mathrm{C} 5-\mathrm{C} 6-\mathrm{N} 1$ & $55.8(3)$ & $\mathrm{C} 15-\mathrm{C} 16-\mathrm{C} 17-\mathrm{C} 18$ & $0.0(5)$ \\
\hline $\mathrm{C} 21-\mathrm{C} 5-\mathrm{C} 6-\mathrm{C} 7$ & $161.7(2)$ & $\mathrm{C} 14-\mathrm{C} 13-\mathrm{C} 18-\mathrm{C} 17$ & $-0.8(4)$ \\
\hline $\mathrm{C} 4-\mathrm{C} 5-\mathrm{C} 6-\mathrm{C} 7$ & $-74.5(3)$ & $\mathrm{C} 2-\mathrm{C} 13-\mathrm{C} 18-\mathrm{C} 17$ & $-178.4(2)$ \\
\hline $\mathrm{N} 1-\mathrm{C} 6-\mathrm{C} 7-\mathrm{C} 8$ & $-133.9(2)$ & $\mathrm{C} 16-\mathrm{C} 17-\mathrm{C} 18-\mathrm{C} 13$ & $0.6(4)$ \\
\hline $\mathrm{C} 5-\mathrm{C} 6-\mathrm{C} 7-\mathrm{C} 8$ & $-5.4(4)$ & $\mathrm{O} 1-\mathrm{C} 19-\mathrm{N} 1-\mathrm{C} 6$ & $13.1(3)$ \\
\hline $\mathrm{N} 1-\mathrm{C} 6-\mathrm{C} 7-\mathrm{C} 12$ & $51.0(3)$ & $\mathrm{C} 20-\mathrm{C} 19-\mathrm{N} 1-\mathrm{C} 6$ & $-166.3(2)$ \\
\hline $\mathrm{C} 5-\mathrm{C} 6-\mathrm{C} 7-\mathrm{C} 12$ & $179.5(2)$ & $\mathrm{O} 1-\mathrm{C} 19-\mathrm{N} 1-\mathrm{C} 2$ & $177.7(2)$ \\
\hline $\mathrm{C} 12-\mathrm{C} 7-\mathrm{C} 8-\mathrm{C} 9$ & $0.3(4)$ & $\mathrm{C} 20-\mathrm{C} 19-\mathrm{N} 1-\mathrm{C} 2$ & $-1.7(3)$ \\
\hline $\mathrm{C} 6-\mathrm{C} 7-\mathrm{C} 8-\mathrm{C} 9$ & $-174.8(3)$ & $\mathrm{C} 7-\mathrm{C} 6-\mathrm{N} 1-\mathrm{C} 19$ & $-108.2(2)$ \\
\hline $\mathrm{C} 7-\mathrm{C} 8-\mathrm{C} 9-\mathrm{C} 10$ & $0.1(5)$ & $\mathrm{C} 5-\mathrm{C} 6-\mathrm{N} 1-\mathrm{C} 19$ & $119.4(2)$ \\
\hline $\mathrm{C} 8-\mathrm{C} 9-\mathrm{C} 10-\mathrm{C} 11$ & $-0.3(5)$ & $\mathrm{C} 7-\mathrm{C} 6-\mathrm{N} 1-\mathrm{C} 2$ & $86.6(2)$ \\
\hline $\mathrm{C} 9-\mathrm{C} 10-\mathrm{C} 11-\mathrm{C} 12$ & $0.1(5)$ & $\mathrm{C} 5-\mathrm{C} 6-\mathrm{N} 1-\mathrm{C} 2$ & $-45.8(2)$ \\
\hline $\mathrm{C} 10-\mathrm{C} 11-\mathrm{C} 12-\mathrm{C} 7$ & $0.4(5)$ & $\mathrm{C} 13-\mathrm{C} 2-\mathrm{N} 1-\mathrm{C} 19$ & $64.0(3)$ \\
\hline $\mathrm{C} 8-\mathrm{C} 7-\mathrm{C} 12-\mathrm{C} 11$ & $-0.6(4)$ & $\mathrm{C} 3-\mathrm{C} 2-\mathrm{N} 1-\mathrm{C} 19$ & $-173.40(18)$ \\
\hline $\mathrm{C} 6-\mathrm{C} 7-\mathrm{C} 12-\mathrm{C} 11$ & $174.8(2)$ & $\mathrm{C} 13-\mathrm{C} 2-\mathrm{N} 1-\mathrm{C} 6$ & $-131.6(2)$ \\
\hline $\mathrm{N} 1-\mathrm{C} 2-\mathrm{C} 13-\mathrm{C} 14$ & $40.9(3)$ & $\mathrm{C} 3-\mathrm{C} 2-\mathrm{N} 1-\mathrm{C} 6$ & $-8.9(3)$ \\
\hline $\mathrm{C} 3-\mathrm{C} 2-\mathrm{C} 13-\mathrm{C} 14$ & $-81.8(3)$ & & \\
\hline
\end{tabular}

Hydrogen-bond geometry $\left(A,{ }^{\circ}\right)$

\begin{tabular}{lllll}
\hline$D-\mathrm{H} \cdots A$ & $D-\mathrm{H}$ & $\mathrm{H} \cdots A$ & $D \cdots A$ & $D-\mathrm{H} \cdots A$ \\
\hline $\mathrm{C} 20-\mathrm{H} 20 B \cdots \mathrm{O} 1^{\mathrm{i}}$ & 0.96 & 2.44 & $3.292(3)$ & 148 \\
\hline
\end{tabular}

Symmetry code: (i) $-x+1, y+1 / 2,-z+1$. 